\title{
Variación temporal de la morfología funcional del fitoplancton en una planicie inundable del Caribe Colombiano
}

\section{Temporal variation of phytoplankton functional morphology in a Colombian Caribbean floodplain system}

\author{
Ana María Zabala Agudelo ${ }^{\circledR}$, Esnedy Hernández ${ }^{\circledR}$, Diana María Agudelo ${ }^{\circledR}$, Nestor Jaime \\ Aguirre ${ }^{\circledR}$, Fabio de Jesús Vélez ${ }^{\circledR}$
}

\section{Resumen}

Analizamos la variación temporal de los grupos funcionales basados en la morfología del fitoplancton (GFBM) en el sistema inundable de Ayapel a través de la comparación de dos muestreos previos realizados entre los 2005 y 2007 y uno nuevo hecho en el 2015. Se estimó el biovolumen $\left(\mathrm{mm}^{3} .1^{-1}\right)$ y los rasgos morfológicos como volumen, relación superficie/volumen (S/V) y dimensión linear máxima (DLM). El análisis temporal fue realizado acorde con los niveles de precipitación pluvial, y mediante un análisis de agrupamiento se definieron dos períodos previos (época seca y época de transición) y uno nuevo correspondiente al año 2015 (msENSO: época seca con influencia ENSO). En las épocas seca y de transición, el grupo predominante fue el GFBM III con altas DLM $(108.77 \mu \mathrm{m})$ y relaciones $\mathrm{S} / \mathrm{V}(1.44 \mu \mathrm{m})$, mientras que en el msENSO se observaron bajos biovolumenes $(0.016$ $\mathrm{mm}^{3} .1^{-1}$ ) y la predominancia del GFBM V. La comparación de la morfología funcional entre los años evidenció la transición de formas filamentosas a formas flageladas del fitoplancton, permitiéndole un mejor ajuste ante las presiones ambientales del área de estudio.

Palabras clave. Biovolumen. Fitoplancton. Inundación y sequía. Rasgos morfológicos.

\begin{abstract}
We analyzed temporal variation of morphology-based functional groups of phytoplankton (MBFG) in the Ayapel floodplain system, through the comparison of two previous samplings carried out between 2005 and 2007 and a new one made in 2015. The biovolume $\left(\mathrm{mm}^{3} \cdot 1^{-1}\right)$ and morphological features such as volume, surface area: volume ratio (S/V) and maximum linear dimension (MLD) were performed. Temporal analysis was carried out according to pluvial precipitation levels, and by means of a grouping analysis two previous periods (dry season and transition period) and a new one corresponding to 2015 (msENSO: dry season with ENSO influence) were defined. In the dry and transition seasons, the predominant group was GFBM III with high MLD (108.77 $\mu \mathrm{m})$ and S/V ratios $(1.44 \mu \mathrm{m})$, while in msENSO low biovolumes $\left(0.016 \mathrm{~mm}^{3} .1^{-1}\right)$ and the predominance of GFBM V were observed. The comparison of functional morphology between years showed a transition from filamentous forms to flagellated forms of phytoplankton, allowing a better fit to the environmental pressure of the study area.
\end{abstract}

Keywords. Biovolume. Flood and drought. Morphological features. Phytoplankton. 


\section{Introducción}

El empleo de las estrategias funcionales es un concepto inicialmente propuesto por Grime (1979) con base en estudios de plantas y posteriormente fue transferido y adaptado al fitoplancton por Reynolds (1980). Luego, Reynolds et al. (2002), introdujeron a la clasificación funcional asociaciones entre la morfología del fitoplancton y las condiciones ambientales.

Las estrategias morfofuncionales son definidas por Margalef (1993) como la respuesta de las formas del fitoplancton frente a las características ambientales, y donde la coocurrencia de los organismos depende de las diferencias en los procesos biológicos, crecimiento, estrategias de adquisición de recursos, capacidad de inhibir los procesos de pérdida, regulación de la flotabilidad y migración vertical (Mihaljevic et al., 2015).

La clasificación morfofuncional empleada en el presente estudio es la propuesta por Kruk et al. (2010), la cual resume las características funcionales y la variabilidad morfológica de los organismos en siete grupos funcionales (GFBM I-VII) que reflejan las diferentes habilidades para absorber luz y nutrientes, destrezas en el crecimiento y mecanismos para prevenir la sedimentación y la depredación (Pacheco et al., 2010; tabla 1). Además, considera la interacción entre las características limnológicas del cuerpo de agua y el condicionamiento de las formas biológicas, definiendo tolerancias y vulnerabilidades en el ambiente, ya sea desde los rasgos numéricos basados en el tamaño, como la dimensión linear máxima (DLM), el volumen $(\mathrm{V})$, la relación superficie volumen (S/V) o por rasgos especializados como aerótopos (Aer), flagelos (Fla), mucílago (Muc), heterocitos (Het) y sílice (Si). Dicha agrupación resulta en que determinados grupos de especies pueden predominar en un momento particular, compartiendo rasgos morfológicos y garantizando un mejor ajuste a las condiciones ambientales del sistema. Constituyen así un soporte para el monitoreo de los ecosistemas acuáticos, por medio de la predicción de la composición de la comunidad y su relación con las condiciones ambientales (Kruk et al., 2011; Kruk \& Segura, 2012).

Diversos autores han concluido que el empleo de la morfología funcional permite revelar cambios asociados a la ecología del fitoplancton en ecosistemas acuáticos con diferentes tensores y características ambientales (Lins et al., 2017; Segura et al., 2018; Amorim et al., 2019; Kruk et al., 2017; Muñoz-López et al., 2017). Algunos estudios realizados en sistemas inundables han sido desarrollados en el lago de inundación brasilero 'Batata' y en la planicie de inundación del rio Danubio, los cuales han concluido que las propiedades físicas del medio, así como su dinámica pulsátil son altamente determinantes sobre las estrategias o asociaciones de los grupos funcionales encontrados (Mihaljević et al., 2015; Muñoz-López et al., 2017). En lagos de inundación con una columna de agua mezclada, somera y turbia, como la Ciénaga de Ayapel, predominan organismos de mayor tamaño, altos volumen y razón $\mathrm{S} / \mathrm{V}$, adaptados a muchas perturbaciones físicas y tolerantes a poca luz (Hernández-Atilano et al., 2012).

Esta planicie de inundación es un ecosistema estratégico debido a los servicios ecosistémicos que ofrece, como la regulación hidrológica de los ríos San Jorge y Cauca, así como el control del microclima, la mitigación de inundaciones, sustento alimentario, transporte fluvial, turismo y recreación (Aguilera, 2011), y fue declarado Distrito de Manejo Integrado en el 2009 y posteriormente sitio Ramsar en el 2018. Presenta también una serie de tensores ambientales como el aprovechamiento forestal, la deforestación para la adecuación de potreros destinados a la ganadería, la sobrepesca y captura de peces por debajo de las tallas mínimas, la explotación de fauna y flora endémicas y la contaminación del agua producto de las actividades agrícolas y ganaderas. Así mismo, la práctica de minería extensiva e informal de oro y níquel que se realiza sobre las cuencas de los ríos San Jorge y Cauca (García et al., 2010), inciden en la calidad ecológica, la conectividad y la biodiversidad acuática y terrestre del ecosistema (Junk \& Wantzen, 2004). 
Adicionalmente, el pulso de la ciénaga ha estado influenciado por fenómenos naturales como La Niña, en 2010 (CEPAL, 2012) y El Niño, en 2015 (IDEAM, 2015); y por factores antrópicos como el rompimiento de un dique en el río Cauca en 2010, que generó un aumento prolongado del nivel medio de la ciénaga (Correa, 2013).

Bajo dicho contexto, la pregunta de éste estudio es ¿tienen incidencia los tensores ambientales que se presentan en la ciénaga de Ayapel sobre la expresión de la ecología funcional del fitoplancton?

Se plantea como hipótesis que, si la morfología funcional está condicionada por las características limnológicas y éstas a su vez por los aspectos tensionantes a los que se ha sometido el ecosistema, entonces el uso de la clasificación morfofuncional permitirá identificar las estrategias ecológicas que los organismos han adecuado para lograr un mejor desempeño, en respuesta a los cambios en el entorno.

Tabla 1. Características morfológicas de los grupos funcionales de fitoplancton basados en la morfología, su filiación tax onómica, el valor medio y los rangos de variación de los rasgos morfológicos como volumen (V), superficie (S), relación superficie volumen (S/V), dimensión linear máxima (DLM) y de los rasgos especializados como aerótopos (Aer), flagelos (Fla), mucílago (Muc), heterocitos (Het) y sílice (Si). Modificado de Kruk et al. (2010).

\begin{tabular}{|c|c|c|c|c|c|c|c|c|c|c|c|}
\hline \multirow[b]{2}{*}{ Grupo } & \multirow[b]{2}{*}{ Descripción } & \multirow[b]{2}{*}{$\begin{array}{c}\text { Grupos } \\
\text { taxonómicos }\end{array}$} & \multicolumn{4}{|c|}{ Rasgos morfológicos } & \multicolumn{5}{|c|}{ Rasgos especializados } \\
\hline & & & $\begin{array}{c}\mathrm{V} \\
\mu \mathrm{m}^{3}\end{array}$ & $\begin{array}{c}S \\
\mu \mathrm{m}^{2}\end{array}$ & $\begin{array}{l}\mathrm{S} / \mathrm{V} \\
\mu \mathrm{m}^{-1}\end{array}$ & $\begin{array}{c}\text { DLM } \\
\mu \mathrm{m}\end{array}$ & Aer & Fla & Muc & Het & Si \\
\hline I & Pequeños, & Chlorococcales, & 12.9 & 134 & 5.1 & 7.8 & 0.02 & 0.03 & 0.06 & 0.00 & 0.00 \\
\hline 8 & $\begin{array}{c}\text { alta S/V: } \\
\text { unicelulares y } \\
\text { algunas } \\
\text { pequeñas } \\
\text { colonias o } \\
\text { filamentos }\end{array}$ & $\begin{array}{c}\text { Chroococcales } \\
\text { (pico y } \\
\text { nanoplacton), } \\
\text { Oscillatoriales, } \\
\text { Xanthophyceae, } \\
\text { Ulothricales }\end{array}$ & $\begin{array}{l}0.3 \\
-120\end{array}$ & $\begin{array}{c}2.3 \\
-8191\end{array}$ & $\begin{array}{c}1.5 \\
-28.7\end{array}$ & $\begin{array}{c}0.8 \\
-43.6\end{array}$ & $0-1$ & $0-1$ & $0-1$ & 0 & 0 \\
\hline II & Chrysophytaes, & & 626 & 308 & 1.6 & 17.1 & 0.00 & 1.00 & 0.00 & 0.00 & 1.00 \\
\hline & $\begin{array}{c}\text { Estructuras } \\
\text { silíceas con } \\
\text { exoesqueleto y } \\
\text { flagelo }\end{array}$ & Chrysophytaes & $\begin{array}{c}1.0 \\
-10469\end{array}$ & $\begin{array}{c}4.7 \\
-2783\end{array}$ & $\begin{array}{l}0.3 \\
-6.8\end{array}$ & $\begin{array}{c}2.0 \\
-164\end{array}$ & 0 & 1 & 0 & 0 & 1 \\
\hline III & Filamentos & & 1541 & 1553 & 1.7 & 95.7 & 1.00 & 0.00 & 0.27 & 0.64 & 0.00 \\
\hline & $\begin{array}{c}\text { grandes de } \\
\text { Cyanobacteria } \\
\text { con aerótopos } \\
\text { y heterocistos }\end{array}$ & $\begin{array}{c}\text { Nostocales, } \\
\text { Oscillatoriales }\end{array}$ & $\begin{array}{c}8.1 \\
-8708\end{array}$ & $\begin{array}{c}19.6 \\
-4598\end{array}$ & $\begin{array}{l}0.4 \\
-4.3\end{array}$ & $\begin{array}{c}2.5 \\
-259\end{array}$ & 1 & 0 & $0-1$ & $0-1$ & 0 \\
\hline IV & Grandes & Chlorococcales, & 1543 & 791 & 1.6 & 37.0 & 0.00 & 0.00 & 0.00 & 0.00 & 0.00 \\
\hline$\alpha$ & $\begin{array}{l}\text { unicelulas, } \\
\text { colonias y } \\
\text { filamentos }\end{array}$ & $\begin{array}{c}\text { Oscillatoriales, } \\
\text { Xanthophyceae, } \\
\text { Zygnematophyceae }\end{array}$ & $\begin{array}{c}12.7 \\
-48255\end{array}$ & $\begin{array}{c}29.1 \\
-18200\end{array}$ & $\begin{array}{c}0.1 \\
-5.1\end{array}$ & $\begin{array}{l}4.6 \\
-700\end{array}$ & 0 & 0 & 0 & 0 & 0 \\
\hline
\end{tabular}




\begin{tabular}{|c|c|c|c|c|c|c|c|c|c|c|c|}
\hline \multirow[t]{2}{*}{ V } & \multirow[b]{2}{*}{$\begin{array}{l}\text { Flagelados } \\
\text { unicelulares }\end{array}$} & \multirow{2}{*}{$\begin{array}{c}\text { Cryptophytaes, } \\
\text { Dinoflagellataes, } \\
\text { Euglenophytaes, } \\
\text { Volvocales, } \\
\text { Chlorococcales }\end{array}$} & 2444 & 764 & 1.2 & 17.5 & 0.00 & 1.00 & 0.00 & 0.00 & 0.00 \\
\hline & & & $\begin{array}{c}2.4 \\
-164779\end{array}$ & $\begin{array}{c}8.9 \\
-20997\end{array}$ & $\begin{array}{c}0.1 \\
-4.9\end{array}$ & $\begin{array}{c}2.1 \\
-190\end{array}$ & 0 & 1 & 0 & 0 & 0 \\
\hline \multirow[t]{2}{*}{ VI } & \multirow{2}{*}{$\begin{array}{c}\text { Diatomeas no } \\
\text { flageladas, con } \\
\text { exoesqueleto } \\
\text { de sílice }\end{array}$} & \multirow{2}{*}{$\begin{array}{c}\text { Diatomeas } \\
\text { Centrales y } \\
\text { Pennales }\end{array}$} & 3143 & 1344 & 1.1 & 44.1 & 0.00 & 0.00 & 0.00 & 0.00 & 1.00 \\
\hline & & & $\begin{array}{c}7.8 \\
-57106\end{array}$ & $\begin{array}{c}19.2 \\
-17473\end{array}$ & $\begin{array}{c}0.2 \\
-3.6\end{array}$ & $\begin{array}{c}2.8 \\
-244\end{array}$ & 0 & 0 & 0 & 0 & 1 \\
\hline \multirow[t]{2}{*}{ VII } & \multirow{2}{*}{$\begin{array}{c}\text { Colonias, } \\
\text { algunas de } \\
\text { gran tamaño } \\
\text { con mucílago o } \\
\text { lípidos }\end{array}$} & \multirow{2}{*}{$\begin{array}{c}\text { Chlorococcales, } \\
\text { Chroococcales, } \\
\text { algunas } \\
\text { Oscillatoriales }\end{array}$} & 43152 & 3062 & 1.0 & 30.1 & 0.04 & 0.00 & 1.00 & 0.00 & 0.00 \\
\hline & & & $\begin{array}{c}10.9 \\
-2.4 \mathrm{E} 6\end{array}$ & $\begin{array}{c}24 . \\
-87616\end{array}$ & $\begin{array}{c}0 \\
-6.0\end{array}$ & $\begin{array}{c}3.5 \\
-244\end{array}$ & $0-1$ & 0 & 1 & 0 & 0 \\
\hline
\end{tabular}

\section{Materiales y métodos}

Área de estudio. El sistema cenagoso de Ayapel está localizado en el noroccidente de Colombia, en la cuenca media inferior del río San Jorge, a $25 \mathrm{~km}$ de la zona minera con la más alta producción de oro en el país (sur de Bolívar y noreste de Antioquia). Es parte de la planicie aluvial de la depresión Momposina, sobre la cual confluyen los ríos Cauca, Magdalena y San Jorge (Zapata et al., 2013). Comprende un cuerpo de agua principal y varios cuerpos menores conectados a través de una red de caños de variable magnitud (CVS, 2014). Según el sistema de clasificación de tipos de humedales de la Convención Ramsar, es un complejo de humedales continentales que forma parte del macrosistema de humedales y zonas anegables de la depresión Momposina (Figura 1).

De acuerdo con la clasificación del sistema de Holdridge, la ciénaga corresponde a un ecosistema de bosque húmedo tropical. La elevación media es de 22 m s.n.m., el promedio anual de lluvias fluctúa entre 2000 y $2500 \mathrm{~mm}$, con una temperatura ambiente promedio anual superior a los $25^{\circ} \mathrm{C}$ (Aguirre et al., 2005; Rangel-Ch. \& Arellano, 2010). El régimen de precipitación está enmarcado en una época de lluvias de abril a noviembre y una época seca de diciembre a marzo, aasociado al desplazamiento latitudinal de la zona de convergencia intertropical
(Aguilera, 2009). Así mismo, se presentan dos periodos de transición de aguas bajas en ascenso entre abril y mayo y de aguas altas en descenso entre diciembre y enero.

Diseño del muestreo. Con el fin de obtener el mayor contraste temporal posible, se analizó la información de dos investigaciones previas hechas entre 2005 y 2007; adicionalmente se incluye un muestreo realizado en 2015, influenciado por el fenómeno Niño-Oscilación del Sur (ENSO). Las investigaciones previas incluyeron el análisis de variables limnológicas, así como variables morfológicas del fitoplancton (Hernández et al., 2008, 2012).

Para el monitoreo del 2015 se realizaron tres muestreos, en septiembre, octubre y diciembre, en siete estaciones ubicadas en el espejo de agua principal del sistema y correspondientes con la localización y método de muestreo de las estaciones incluidas en las investigaciones previas.

A partir de los niveles de precipitación mensuales y por medio de un análisis de conglomerados con el método Ward, se establecieron tres épocas hidrológicas correspondientes a bajas precipitaciones (promedio 42 $\mathrm{mm})$ : época seca $(\mathrm{mS})$; transición a lluvias (promedio $350 \mathrm{~mm}$ ): época de transición (mT); y bajas precipitaciones con influencia ENSO (promedio $54 \mathrm{~mm}$ ): msENSO, 


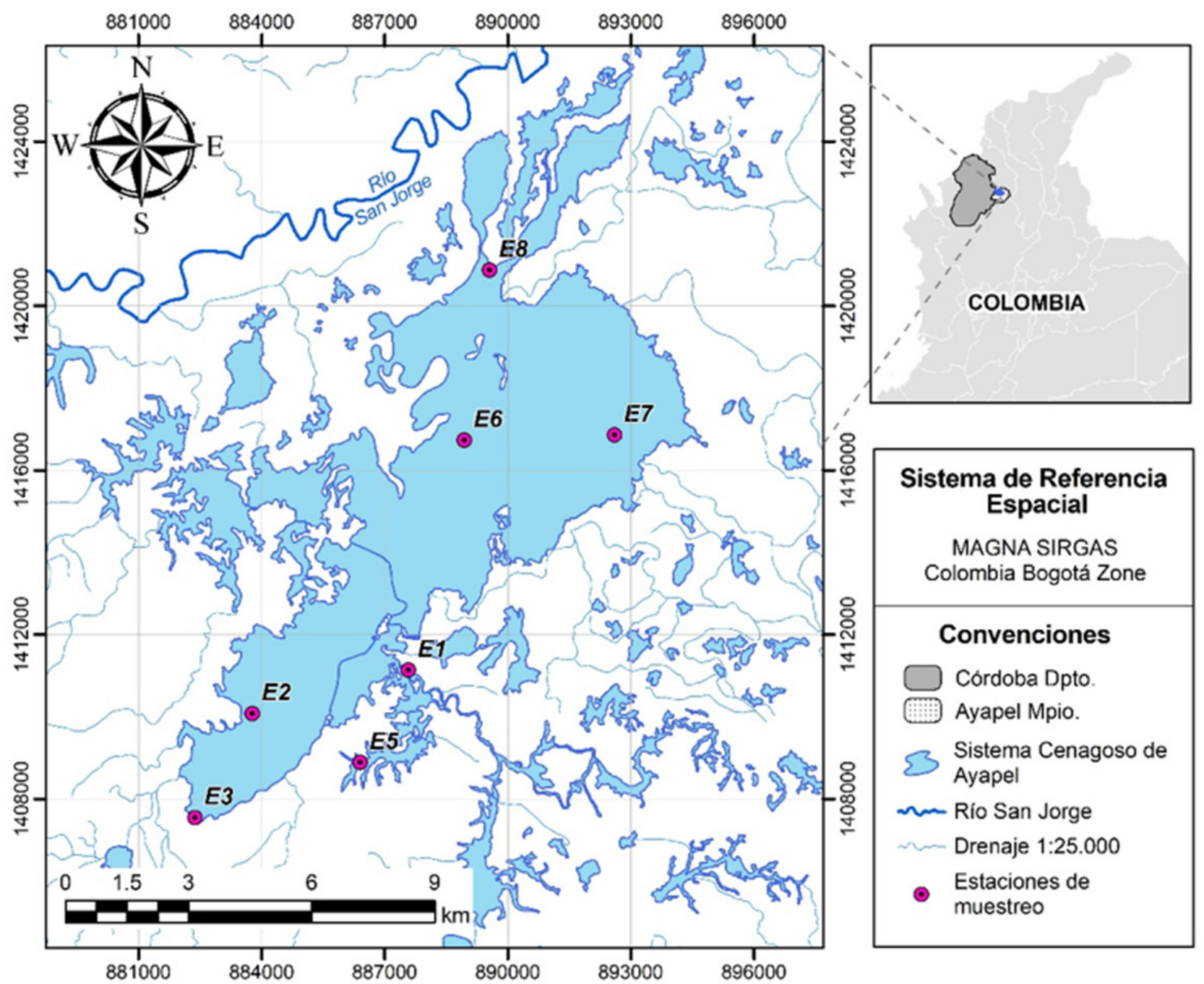

Figura 1. Ubicación geográfica de la Ciénaga de Ayapel y de las estaciones de muestreo (Córdoba, Colombia).

la cual agrupó todos los muestreos realizados durante el 2015.

Los datos de precipitación pluvial correspondientes a los meses de los muestreos fueron obtenidos del banco de datos del Instituto de Hidrobiología y Meteorología y Estudios Ambientales (IDEAM, 2015) de la regional Atlántico. Las estaciones meteorológicas consideradas fueron Cecilia y Ayapel.

Análisis de las variables ambientales. Por medio de un equipo multiparamétrico HACH HQ40d se midieron in situ la temperatura del agua $\left({ }^{\circ} \mathrm{C}\right)$, el oxígeno di- suelto $\left(\mathrm{mg} . \mathrm{l}^{-1}\right)$, el $\mathrm{pH}$, el potencial redox $(\mathrm{mV})$ y la conductividad eléctrica $(\mu \mathrm{S} / \mathrm{cm})$; adicionalmente, con un disco Secchi se midió la transparencia (m). Por su parte, las variables de turbiedad (FNU) y alcalinidad (mg.l ${ }^{-1}$ ) se midieron únicamente durante el 2015 (m2015) por medio de un Turbidímetro y un Test Visocolor, respectivamente.

Para la determinación de las concentraciones de ortofosfatos $\left(\mathrm{PO}_{4}^{3-}\right)$, nitratos $\left(\mathrm{NO}_{3}\right)$, amonio $\left(\mathrm{NH}_{4}^{+}\right)$, dureza total $\left(\mathrm{mg} . \mathrm{l}^{-1}\right)$ y alcalinidad $\left(\mathrm{mg} . \mathrm{l}^{-1}\right)$ se tomaron en cada una de las siete estaciones, muestras integradas en la columna de agua por medio de una botella tipo 
Kemmerer. Las concentraciones de nutrientes fueron establecidas con un fotómetro compacto PF-12, mientras que la dureza total y la alcalinidad fueron determinadas a través de un Test Visocolor.

Análisis del fitoplancton. La determinación y conteo del fitoplancton se realizó en un microscopio invertido con un aumento de 400X, provisto de un software para la medición y toma de fotos de los organismos. Cada muestra fue tratada y cuantificada siguiendo la técnica de Utermohl (1958).

El volumen (V), la superficie (S) y la dimensión linear máxima (DLM) de cada organismo se determinaron por medio de las ecuaciones geométricas propuestas por Hillebrand et al. (1999). El biovolumen individual $\left(\mu \mathrm{m}^{3} .1^{-1}\right)$ se estimó multiplicando el volumen $\left(\mu \mathrm{m}^{3}\right)$ por la densidad de individuos (ind. $\mathrm{l}^{-1}$ ). En cada una de las muestras recolectadas se midieron hasta10 individuos de cada morfotipo para obtener las medidas promedio de DLM, S y V.

La clasificación de los organismos en los grupos funcionales basados en la morfología (GFBM) se realizó a partir de las dimensiones morfológicas medias y de la presencia de rasgos especializados de cada taxón, siguiendo la clave dicotómica de Kruk et al. (2010).

Análisis estadístico. Se realizó un análisis descriptivo con medidas de tendencia central y dispersión para las variables ambientales, biovolumen y rasgos morfológicos. Para determinar diferencias estadísticamente significativas a nivel espacial y temporal, y debido a la ausencia de normalidad en los datos, se aplicó la prueba de Kruskal-Wallis, empleando el software Statgraphics Centurion.

Con el objetivo de determinar el modelo estadístico que mejor se ajusta a la respuesta de la variación de los GFBM con respecto a las variables ambientales, se hizo un análisis de correspondencia sin tendencia (DCCA), el cual estima el gradiente ambiental de acuerdo con la respuesta del conjunto de variables biológicas. Dado que la longitud del gradiente ambiental fue de 1.56 des- viaciones estándar, se hizo un análisis de redundancia canónica (RDA).

Todas las variables incluidas en el RDA registraron un factor de inflación menor a 20 y sólo los ortofosfatos y la profundidad total mostraron ser significativas $(\mathrm{p}<0.05)$ para el test de Monte Carlo con 499 permutaciones. Esta prueba se realizó con los datos previamente estandarizados y se empleó el programa de lenguaje de programación $\mathrm{R}$.

\section{Resultados}

Variables ambientales. El análisis de agrupamiento para establecer las épocas hidrológicas definió dos grandes grupos con rangos similares (Figura 2). El primer grupo, correspondiente a menores niveles de precipitación, comprende la época seca $(\mathrm{mS})$ y la época seca con influencia ENSO (msENSO). Por su parte, el segundo grupo abarca las mayores precipitaciones que corresponden a la época de transición (mT).

Las principales diferencias significativas de las variables ambientales entre épocas fueron los rangos de conductividad, las concentraciones de la dureza, los nitratos y los ortofosfatos (Tabla 2). En general las aguas de la ciénaga de Ayapel son aguas blandas, con valores entre 10-25 mg.1-1 $\mathrm{CaCO}_{3}$, a excepción de los muestreos realizados en el 2015, cuyos valores sugieren aguas se-

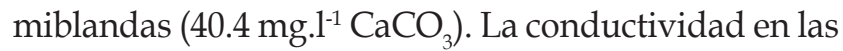
épocas seca y de transición (mS y mT) osciló entre 47-54 $\mu \mathrm{S} . \mathrm{cm}^{-1}$, mientras que en el msENSO el valor medio fue de $112.3 \mu \mathrm{S} . \mathrm{cm}^{-1}$, indicando un aumento en la mineralización de las aguas y por lo tanto en la productividad del sistema (Roldán \& Ramírez, 2008).

Las moderadas concentraciones de oxígeno disuelto (4-6 mg. $\left.\mathrm{l}^{-1}\right)$ corresponden con los rangos de temperatura característica de las regiones, las cuales generan mayor consumo de oxígeno en la columna de agua (Roldán \& Ramírez, 2008). El pH osciló entre 6.8 y 6.9 unidades, mostrando un comportamiento homogéneo y con valores cercanos a la neutralidad. Las concentraciones 
Tabla 2. Estadígrafos de tendencia central de las variables físicas y químicas de la ciénaga de Ayapel, Colombia, por muestreo, teniendo en consideración los registros de las siete estaciones (estudios previos: $\mathrm{mS}$, época seca y $\mathrm{mT}$, época de transición; estudio nuevo 2015: msENSO, época seca con influencia ENSO). Valores p derivados de la prueba de Kruskal-Wallis (valores $\mathrm{p}<0.05$ indican diferencias estadísticamente significativas).

\begin{tabular}{|c|c|c|c|c|c|c|c|c|c|c|c|c|}
\hline 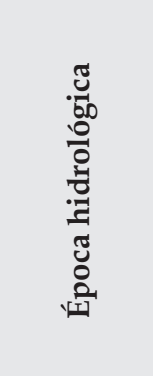 & 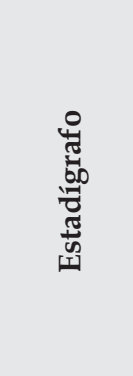 & 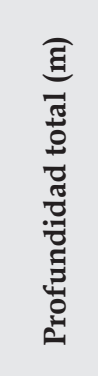 & 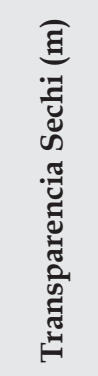 & 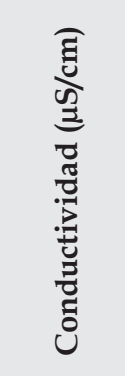 & 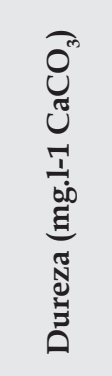 & 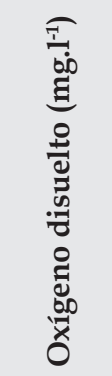 & 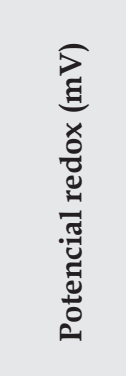 & $\frac{\pi}{2}$ & 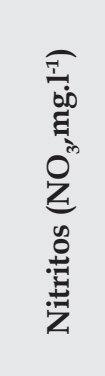 & 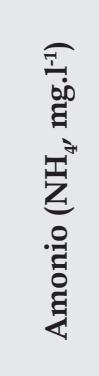 & 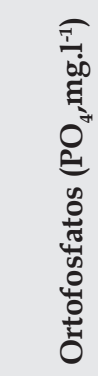 & 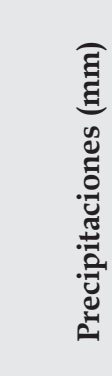 \\
\hline \multirow[t]{3}{*}{$\mathrm{mS}$} & media & 1.903 & 0.211 & 52.956 & 20.077 & 6.639 & 235.923 & 6.854 & 1.054 & 0.195 & 0.361 & 42.33 \\
\hline & desv. & 0.94 & 0.112 & 18.082 & 5.090 & 0.873 & 29.619 & 1.453 & 0.447 & 0.155 & 0.222 & 2.517 \\
\hline & $\mathrm{CV} \%$ & 48.34 & 52.94 & 34.140 & 25.35 & 13.150 & 12.550 & 21.21 & 45.27 & 79.5 & 61.46 & 5.945 \\
\hline \multirow[t]{3}{*}{$\mathrm{mT}$} & media & 3.743 & 0.419 & 47.538 & 22.286 & 4.737 & 226.429 & 6.831 & 0.609 & 0.12 & 0.101 & 362.5 \\
\hline & desv. & 1.061 & 0.227 & 21.611 & 5.736 & 0.803 & 51.117 & 0.418 & 0.781 & 0.059 & 0.096 & 43.493 \\
\hline & CV\% & 28.34 & 54.24 & 45.46 & 25.740 & 16.95 & 22.58 & 6.12 & 128.21 & 49.88 & 95.05 & 11.998 \\
\hline \multirow[t]{3}{*}{ msENSO } & media & 2.623 & 0.58 & 112.308 & 40.417 & 4.618 & & 6.918 & 5.857 & 0.123 & 0.807 & 181.61 \\
\hline & desv. & 1.242 & 0.444 & 49.146 & 17.315 & 1.732 & & 0.251 & 4.832 & 0.064 & 0.390 & 124.985 \\
\hline & CV\% & 47.34 & 76.5 & 43.76 & 42.84 & 37.51 & & 3.62 & 82.51 & 52.43 & 48.34 & 68.82 \\
\hline \multirow[t]{2}{*}{ valor $p$} & época & 0.001 & 0.003 & 0.001 & 0.001 & 0.004 & 0.001 & 0.002 & 0.002 & 0.009 & 0.001 & 0.03 \\
\hline & estación & 0.030 & 0.171 & 0.010 & 0.098 & 0.007 & 0.575 & 0.486 & 0.774 & 0.621 & 0.347 & 0.001 \\
\hline
\end{tabular}




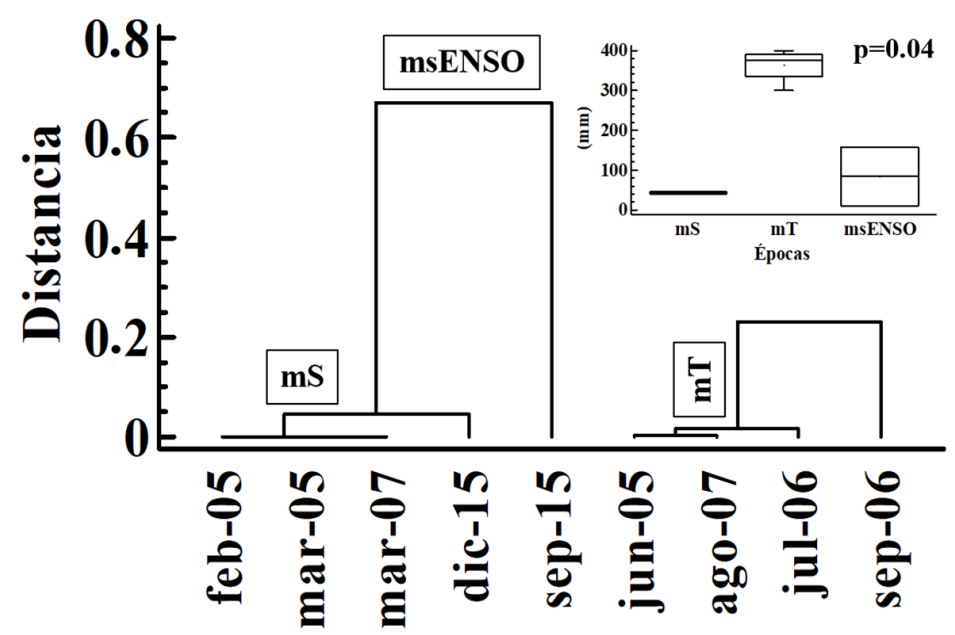

Figura 2. Análisis de conglomerados por el método de Ward de los muestreos de fitoplancton de la ciénaga de Ayapel, Colombia, a partir de los niveles de precipitación, y sus cajas esquemáticas. Valores p obtenidos de la prueba Kruskal-Wallis.

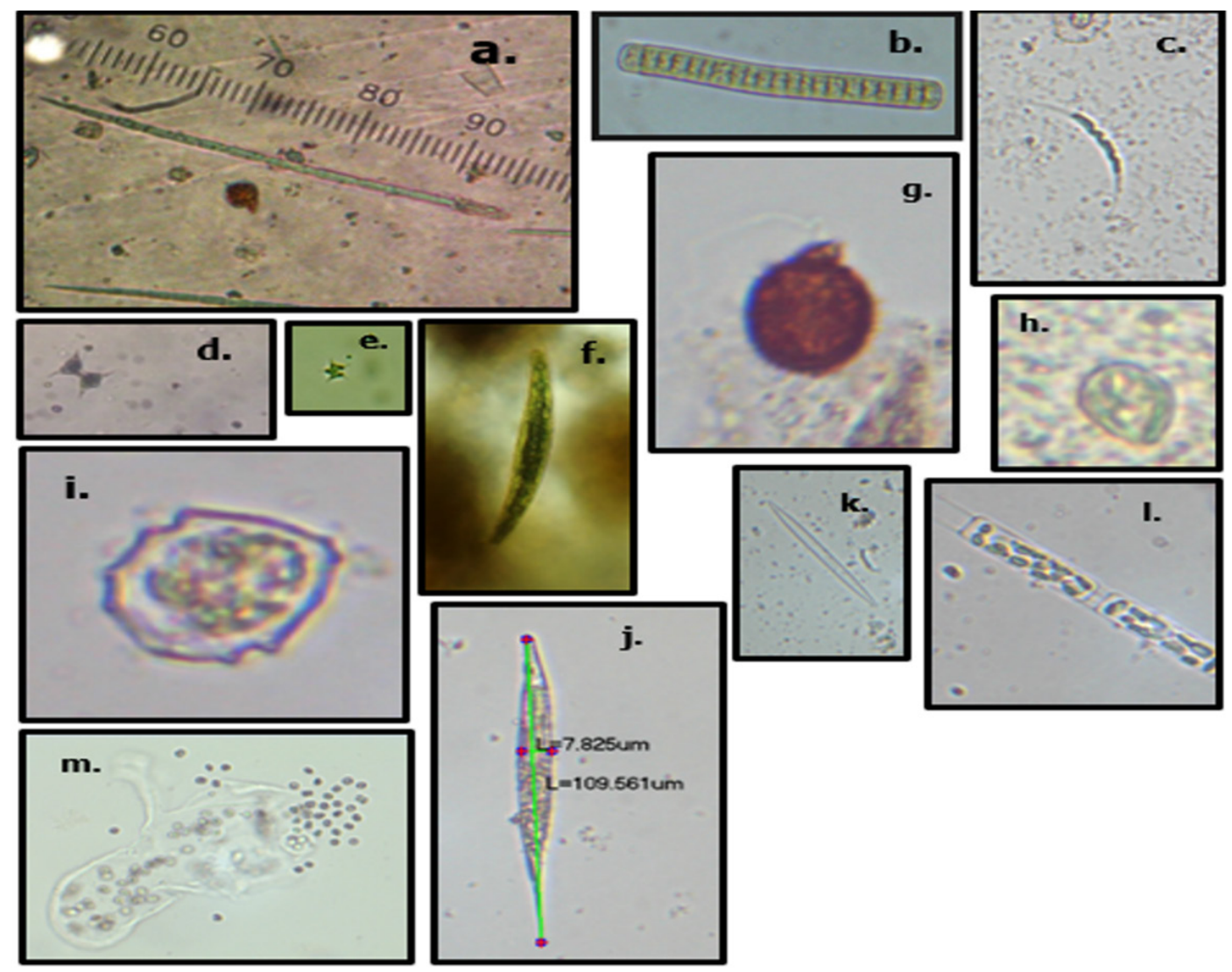

Figura 3. Organismos dominantes en la ciénaga de Ayapel, Colombia, por grupo morfo funcional. GFBM III: a. Cylindrospermopsis sp., b. Oscillatoria sp. GFBM IV: c. Monoraphidium sp., d. Staurastrum sp., e. Tetraedron cf., f. Closterium sp. GFBM V: g. Trachelomonas sp., h. Cryptomonas sp., i. Parvodinium cf., j. Lepocinclis cf. GFBM VI: k. Fragilaria sp., 1. Aulacoseira sp. GFBM VII: m. Aphanocapsa cf. 
medias de los nutrientes en las dos épocas previas se consideran bajas $\left(0.8 \mathrm{mg} . \mathrm{l}^{-1}-\mathrm{NO}^{3}\right)$; no obstante, en el monitoreo del 2015, correspondiente a una época seca con influencia ENSO, se observaron mayores concentraciones de nitratos $\left(5.8 \mathrm{mg} . \mathrm{l}^{-1}\right)$ y ortofosfatos $\left(0.8 \mathrm{mg} . \mathrm{l}^{-1}\right)$.

En relación con la clasificación morfofuncional, se encontraron seis de los siete grupos (GFBM II-VII) propuestos por Kruk et al. (2010) y sus principales variaciones se observaron a nivel temporal. En la figura 3 se muestran los organismos más representativos por grupo morfofuncional. La distribución de los grupos fue la siguiente:

El GFBM II (pequeños flagelados con estructuras silíceas: Chrysophyceae) estuvo presente únicamente en la época de transición (mT), aunque con bajo biovolumen. El GFBM III (grandes filamentos con aerótopos: Cyanobacteria) fue uno de los grupos más importantes en términos de biovolumen y de recurrencia durante el estudio; no obstante, sus mayores valores tuvieron lugar en la época seca (mS). Por su parte el GFBM IV (tamaño medio y ausencia de rasgos especializados) se observó en baja frecuencia y el GFBM V (flagelados unicelulares de tamaño medio a grande) estuvo presente con bajos biovolúmenes en las tres épocas; sin embargo, fue frecuente en todos los muestreos. El GFBM VI (organismos no flagelados y con exoesqueletos de sílice: diatomeas) fue más abundante en las épocas de transición y seca con influencia ENSO, mientras que el GFBM VII (colonias mucilaginosas con lípidos y aerótopos) presentó biomasas muy bajas en las tres épocas de estudio (figura 4).

Los mayores valores de la relación S/V y DLM se observaron en el GFBM III, con valores promedio de 2.66 $\mu \mathrm{m}$ y $263.3 \mu \mathrm{m}$, respectivamente. Dichos valores corresponden a formas pequeñas y filamentosas. En contraste, en el GFBM V se observaron valores promedio de la DLM correspondientes a $50.4 \mu \mathrm{m}$ y una relación S/V de $0.74 \mu \mathrm{m}$; medidas relativamente bajas, correspondientes a morfotipos grandes o deformados. Una alta relación S/V y baja DLM fue observada en el GFBM IV, mientras que en los GFBM VI y VII se observaron valores medios de la relación S/V y bajos de la DLM durante las tres épocas (Figura 5).

Las variaciones de los rasgos morfológicos también se evidenciaron a nivel temporal con respecto a las características de cada grupo (Figura 6). En general, los valores medios, tanto de la relación S/V como de la DLM de los GFBM: II, III, IV, V y VI, disminuyeron notablemente en el muestreo del 2015 (msENSO).

Los resultados del RDA (Figura 7) para explicar la distribución de los seis grupos funcionales basados en la morfología (GFBM: II, III, IV, V, VI, VII), en relación con las variables ambientales (nitratos, conductividad, potencial redox, oxígeno disuelto, dureza total, transparencia, ortofosfatos, profundidad total, amonio, $\mathrm{pH}$ ) generaron un porcentaje de explicación de la varianza total del $61 \%(p=0.02)$. Todos los muestreos correspondientes al año 2015 (msENSO) tuvieron altas concentraciones de ortofosfatos, conductividad, nitratos y dureza y ningún GFBM asociado. Por su parte, a los muestreos previos ( $\mathrm{mS}$ y $\mathrm{mT}$ ) estuvieron relacionados los mayores biovolúmenes de los diferentes GFBM, de los cuales los grupos III y IV estuvieron asociados al potencial redox y el OD. Al msENSO no estuvo asociado ningún GFBM.

\section{Discusión}

La ciénaga de Ayapel corresponde a un sistema oligotrófico con tendencia mesotrófica, según los valores medios de nitritos $\left(5.9 \mathrm{mg}^{-1} \mathrm{l}^{-1} \mathrm{NO}_{3}^{-}\right)$y ortofosfatos $\left(\mathrm{PO}_{4}^{3->} 0.9 \mathrm{mg}^{-1} \mathrm{l}^{-1}\right)$ medidos durante el año 2015 (Esteves, 1998; Vollenweider, 1968). Así mismo, los valores de dureza y turbiedad medidos durante dicho año, están asociados a aguas semiduras $\left(\mathrm{mg} . \mathrm{l}^{-1} \mathrm{CaCO}_{3}>25 \mathrm{mg} \cdot \mathrm{l}^{-1}\right)$ y altos valores de la turbiedad (59.0 FNU).

Los sistemas acuáticos ubicados a bajas elevaciones sobre el nivel del mar tienden a ser naturalmente más turbios por el arrastre de material alóctono y su dinámica pulsátil (Roldán \& Ramírez, 2008). 


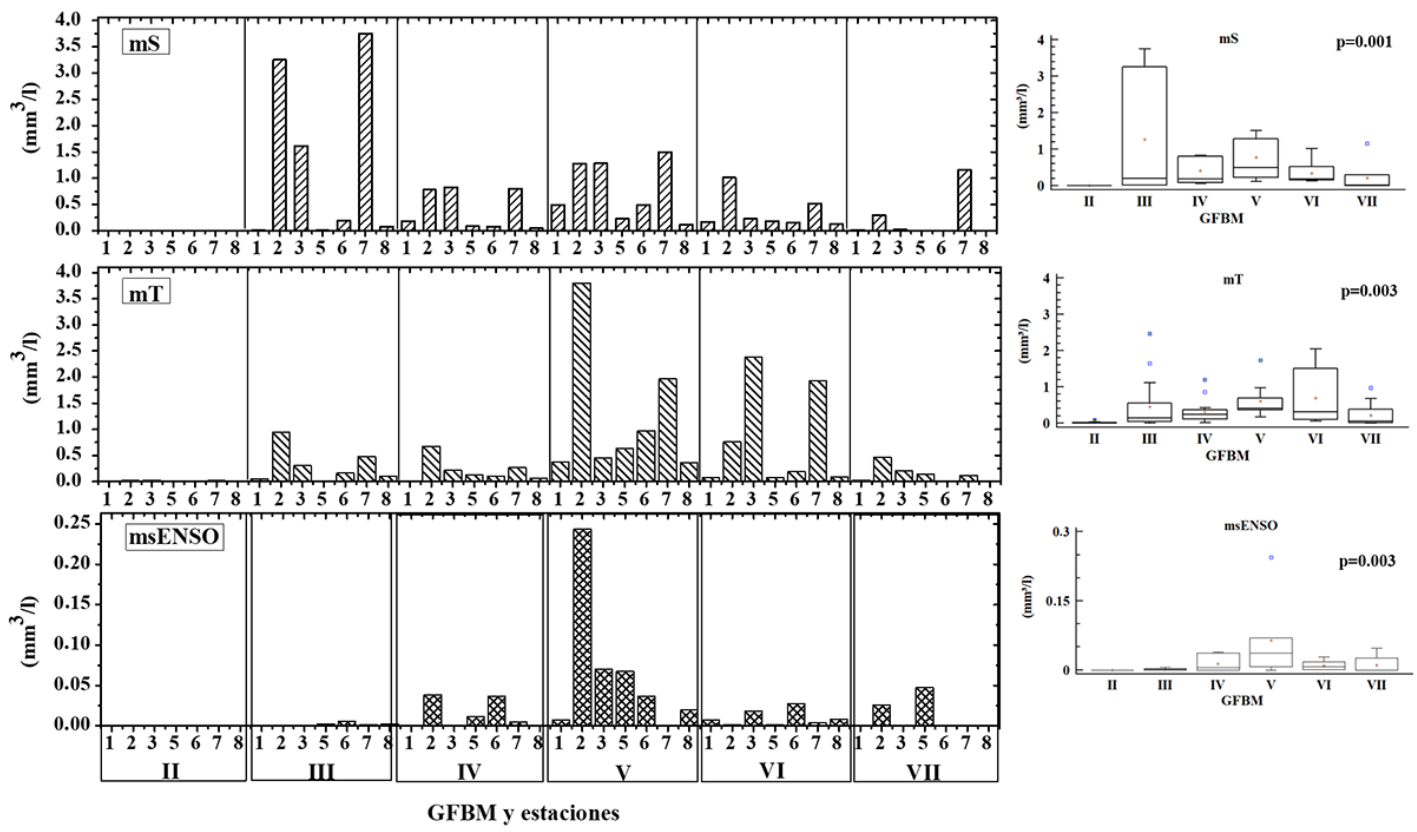

Figura 4. Comparación del biovolumen de los grupos funcionales basados en la morfología para las diferentes épocas de muestreo en la ciénaga de Ayapel, Colombia (estudios previos: mS, época seca y mT, época de transición; estudio nuevo 2015: msENSO. época seca con influencia ENSO). Valores p obtenidos de la prueba Kruskal-Wallis.
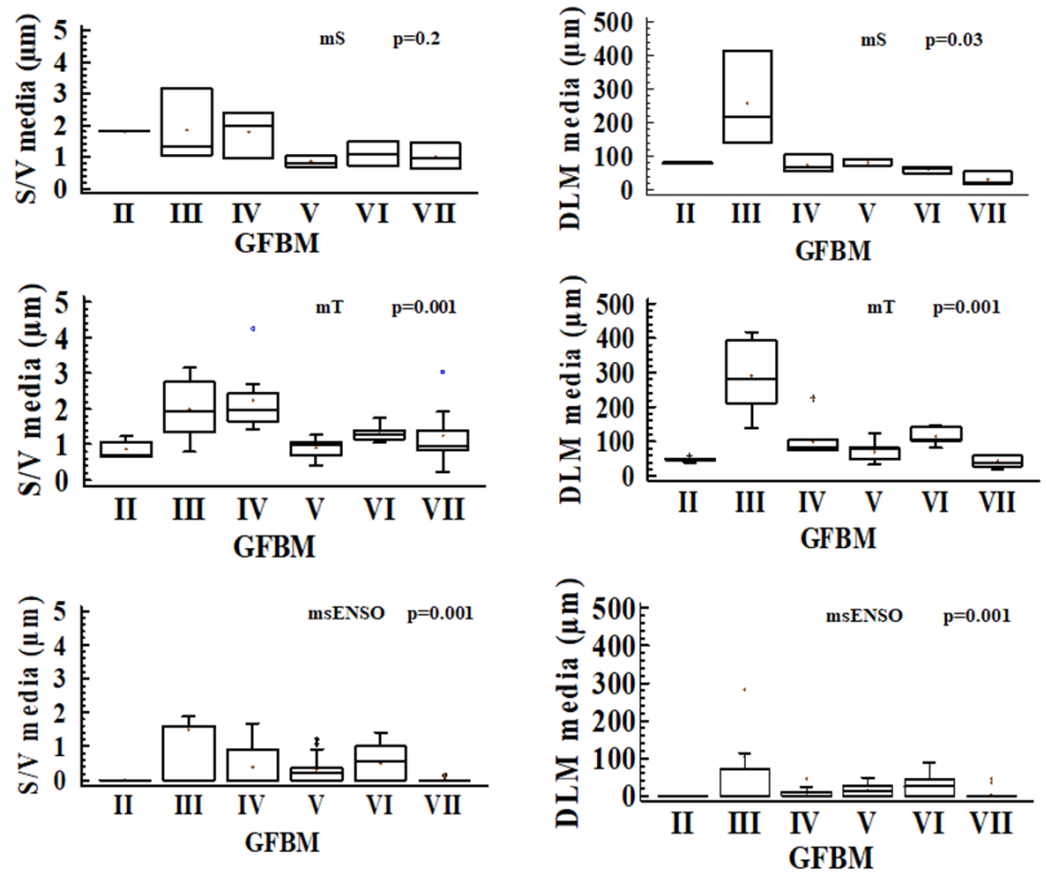

Figura 5. Comparación de los rasgos morfológicos de relación superficie volumen (S/V) y dimensión lineal máxima (DLM) de los grupos morfofuncionales de fitoplancton para las diferentes épocas de muestreo en la ciénaga de Ayapel, Colombia (estudios previos: mS, época seca y mT, época de transición; estudio nuevo 2015: msENSO, época seca con influencia ENSO). Los rasgos presentaron diferencias significativas entre los grupos $(\mathrm{p}<0.05)$. Valores $\mathrm{p}$ derivados de la prueba Kruskal-Wallis. 


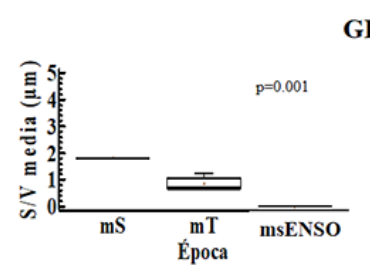

GFBM II
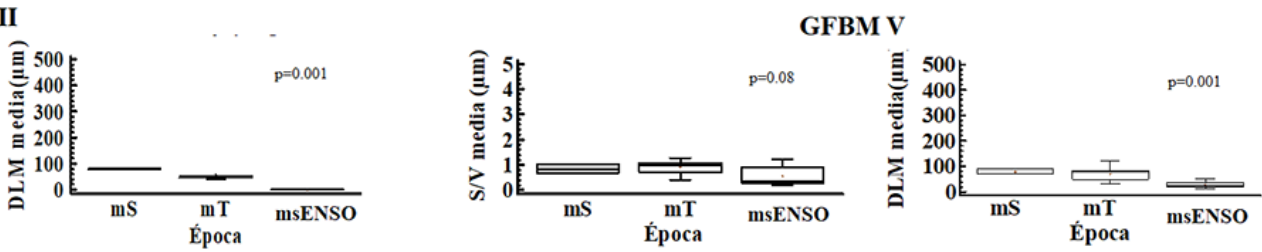

\section{GFBM III}
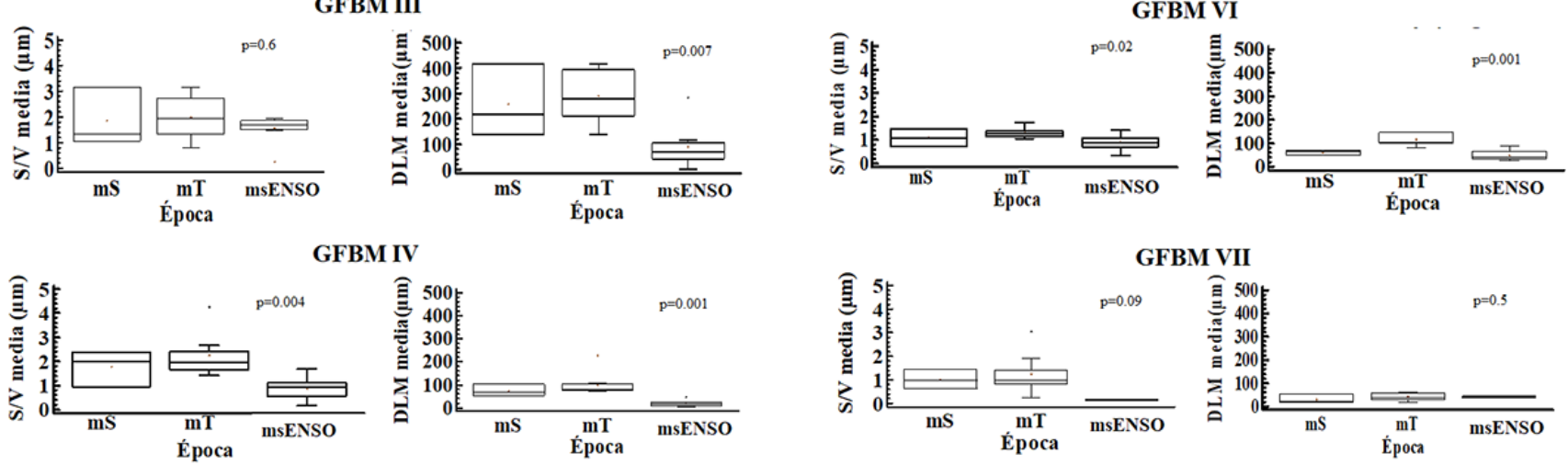

Figura 6. Variación temporal de los rasgos morfológicos de los GFBM II-VI del fitoplancton en la ciénaga de Ayapel, Colombia. Valores p obtenidos de la prueba de Kruskal-Wallis.

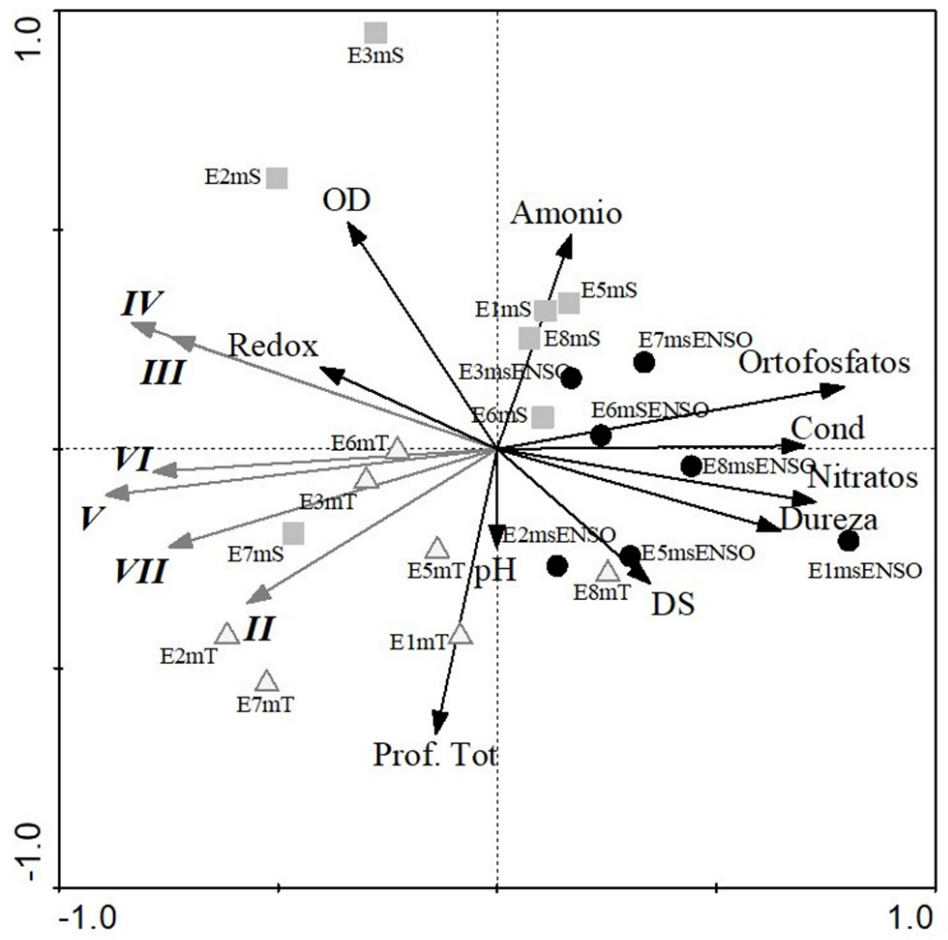

Figura 7. RDA biplot entre épocas, GFBM y variables ambientales para el fitoplancton de la ciénaga de Ayapel, Colombia. Los íconos representan las tres épocas de muestreo (cuadrados: época seca (mS); triángulos: época de transición (mT); círculos: muestreo 2015 (msENSO), las líneas negras simbolizan las variables ambientales y líneas grises los GFBM. 
Sin embargo, desde el año 2010, actividades económicas como la minería y la adecuación de las áreas inundables para diferentes usos han aumentado, lo cual podría incidir en la turbiedad natural del sistema cenagoso de Ayapel.

En general, la turbiedad se relaciona con la productividad de los ecosistemas acuáticos, debido a que interfiere con la transmisión de la luz, aspecto que incide directamente en la productividad primaria del fitoplancton y en el flujo de energía del ecosistema (Amorim et al., 2019). Lo anterior explica la mayor densidad de organismos mixotróficos (GFBM V) durante los muestreos del año 2015, dado que esta cualidad les confiere ventaja en la adaptación frente a unas condiciones poco favorables como la turbiedad, la turbulencia en una columna de agua somera y las variaciones de los nutrientes, factores que habitualmente son limitantes para los organismos estrictamente autótrofos.

De acuerdo con los criterios de Wurts \& Durborow (1992), los valores medios de alcalinidad (0.8 mg. $\left.\mathrm{l}^{-1}\right)$ observados durante los últimos diagnósticos llevados a cabo en el 2015 indican que la ciénaga es bastante vulnerable a posibles cambios bruscos de $\mathrm{pH}$, dada la poca capacidad que tiene para neutralizar el ingreso de compuestos ácidos al sistema.

Según Mihaljević et al. (2015) las condiciones bióticas y abióticas de las planicies de inundación están estrechamente ligadas a los períodos hidroclimáticos. En el presente estudio, la variación de las condiciones físicas y químicas durante el período comprendido resultó en cambios en la distribución temporal de los organismos y biovolumen representado en los diferentes GFBM, lo cual vislumbra cambios importantes en el sistema y en la respuesta de la comunidad del fitoplancton (Muñoz-López et al., 2017).

El biovolumen del fitoplancton en un ecosistema está relacionado con la interacción de varios factores, como sus características fisiológicas y morfológicas, la disponibilidad de nutrientes y luz, la tasa de absorción de nutrientes y la tasa de pérdida de biomasa (Amorim et al., 2019). En el presente estudio, el biovolumen mostró una disminución considerable para el año 2015, para el cual se registraron las mayores concentraciones de nutrientes. Al respecto, Reynolds $(1980,1984,1997)$ argumenta que no sólo la escasez, sino también el exceso de ciertos factores inciden en la adecuación de un organismo; en consecuencia, amplias variaciones en las condiciones ambientales pueden afectar el rango de tolerancia del organismo y su desempeño ecológico.

Los cambios que se evidenciaron en la ciénaga en el 2015 son el resumen de las perturbaciones naturales y antrópicas que se dieron en franjas temporales precedentes y que para este estudio son representadas por la información disponible de los dos estudios previos realizados (época seca y época de transición). De esta forma las comparaciones generadas en los niveles de tolerancia del fitoplancton expresadas a través de las variaciones morfológicas se describen a continuación.

La comunidad fitoplanctónica de la época previa seca estuvo dominado principalmente por la especie Cylindrospermopsis raciborskii, la cual también fue reportada por Jaramillo \& Aguirre (2012) al evaluar la variación temporal del plancton en la ciénaga de Ayapel. C. raciborskii, pertenece al GFBM III, es un representante de los estrategas $r$, presenta baja herbivoría y una alta capacidad de suspensión debido a la presencia de aerótopos (Mur et al., 1999), lo que genera una baja sedimentación y que sus poblaciones sean constantes una vez estén establecidas en un ambiente turbio y turbulento.

La dominancia del GFBM III ha sido reportada en otros lagos tropicales por Bortolini et al. (2016) y Amorim et al. (2019) en ecosistemas eutróficos y someros. Este grupo presenta además una rápida respuesta al estrés ambiental generado por los cambios en el ambiente, los cuales en el caso de la ciénaga de Ayapel corresponden a las perturbaciones naturales de estiaje y llenado del sistema, otorgándoles cierta ventaja en un sistema inundable (Hernández et al., 2008). Las características geométricas de dicho grupo durante la época seca, como un bajo biovolumen y las altas relaciones $\mathrm{S} / \mathrm{V}$ son la respuesta a las limitaciones de luz, sugiriendo que rasgos morfológicos correspondientes a formas 
alargadas y filamentos cilíndricos en la comunidad son el ajuste más eficiente para la captación de luz.

La baja ocurrencia de los GFBM II y IV evidencia el efecto que tiene la turbidez de la ciénaga sobre las adecuaciones morfológicas del fitoplancton, pues éstos grupos requieren de altas transparencias para su desarrollo y en general son favorecidos por condiciones oligotróficas a mesotróficas (Kruk \& Segura, 2012; Izaguirre et al., 2012).

Para la época previa de transición se observó que las especie más abundantes fueron Aulacoseira granulata y Peridinium sp., pertenecientes a los GFBM VI y V, respectivamente. Las especies del género Aulacoseira presentan una amplia variabilidad morfológica intraespecífica, debido a la facultad que tienen para adaptarse a diversas condiciones hidráulicas ( $\mathrm{O}^{\prime}$ Farrell et al., 2001). Respecto al género Peridinium, éstos dinoflagelados no requieren turbulencia para permanecer en las capas superficiales de la columna de agua, debido a su movilidad y a la habilidad de formar quistes que les confiere resistencia a condiciones ambientales desfavorables o cambiantes (Oda \& Bicudo, 2006). Otros rasgos morfológicos que caracterizan al GFBM V, como células de mayor tamaño y un aparato locomotor, les confiere una mayor ventaja adaptativa por poder regular su posición vertical y disminuir la presión por herbivoría (Reynolds, 1997). Adicionalmente, la mayoría de los organismos pertenecientes a este grupo tienen una estrategia ecológica primariamente adquisitiva, denominados estrategas $\mathrm{k}$ y con un crecimiento lento como consecuencia de una relación $\mathrm{S} / \mathrm{V}$ moderada.

Dentro de los seis grupos encontrados, los biovolumenes de los GFBM III y V presentaron mayor variación entre las tres épocas consideradas, con la dominancia del GFBM III en los muestreos previos al año 2015 y la particular prevalencia del GFBM V durante los muestreos del año 2015. La transición del ensamblaje fitoplanctónico de la ciénaga a través de la morfología funcional demuestra que las condiciones ambientales han generado una transformación en la franja de tiempo estudiada, y por lo tanto el empleo de esta metodología fue una herramienta útil para diagnosticar los efectos de los tensores ambientales que se dan en el área de estudio a través del tiempo.

En el muestreo del 2015, el género Trachelomonas, perteneciente al GFBM V, registró los mayores biovolumenes entre todos los muestreos; en contraste, ya no se encontró $C$. raciborskii registrada como dominante en la época previa seca. Al respecto, Garduño et al. (2011) encontraron una importante asociación entre la mayor riqueza de especies de Trachelomonas y el aumento de la turbidez en un cuerpo de agua monomíctico. Este género perteneciente al GFBM V se determina principalmente por la presencia de flagelos, característica que permitiría evadir por medio de la locomoción la baja transparencia en la columna de agua, aspecto que caracterizó los muestreos del año 2015. Es posible entonces que la adecuación morfológica de este grupo, además de beneficiarse por la presencia de flagelo y de la capacidad de desarrollar mixotrofia (Kruk et al., 2010), le otorgue habilidades para tolerar ecológicamente el aumento de la turbiedad y las concentraciones de nutrientes, lo cual podría explicar la permanencia y sobre todo la predominancia del GFBM V durante dicho año.

Los GFBM VI y VII mostraron baja incidencia durante las épocas estudiadas. Al respecto, la baja proporción del GFBM VI se puede explicar por las altas tasas de sedimentación debido a su exoesqueleto silíceo y por la ubicación de las estaciones de muestreo consideradas, ubicadas en el espejo de agua principal lejos de la región litoral, aspectos que no favorecen la presencia de diatomeas perifíticas y meroplanctónicas (Bortolini et al., 2016). Estos mismos autores reportaron que la dominancia del GFBM VII en el Lago Osmar, en el sur de Brasil, estuvo principalmente asociada a la alta disponibilidad de luz, lo que sugiere que la baja presencia de dicho grupo en la ciénaga de Ayapel puede ser el resultado de un efecto fotoinhibidor que tiene la resuspensión de sedimentos en la columna de agua y la consecuente disminución en la transparencia.

La transición de los GFBM y sus rasgos morfológicos sugiere que las condiciones ambientales de la ciénaga 
han presentado cambios en la franja de tiempo considerada y que la adaptación morfológica ha dado paso a la alternancia de grupos con una mayor tolerancia ecológica y estrategias de supervivencia que evaden la alta turbiedad, la turbulencia en la columna de agua y posiblemente la fotoinhibición.

Uno de los principales factores que recientemente han alterado las condiciones de la ciénaga está asociado a las actividades mineras (El Colombiano, 2016). Los efectos de ésta actividad no han sido dimensionados claramente en Ayapel; sin embargo, la resuspensión de sedimentos que dicha actividad implica afecta la productividad del fitoplancton, debido al aumento de sólidos suspendidos y a la forma en la que la luz se atenúa en la columna de agua. En consecuencia, una alta turbiedad resulta en una menor biomasa del fitoplancton debido a las condiciones que impone la limitación de luz (Chaffin et al., 2012).

Dicho factor limitante fue evaluado por Naselli-Flores et al. (2007) en el Lago Arancio, en Sicilia, encontrando que las formas cilíndricas logran desarrollarse bien en un ambiente con disponibilidad de nutrientes y donde la relación de la profundidad de mezcla y la profundidad de la zona eufótica $\left(Z_{\text {mix }} / Z_{\text {eu }^{\prime}} 1.5-3.0\right)$ es baja, ya que para maximizar la exposición de los cloroplastos a la luz y garantizar su recolección, dicha forma alargada es la más eficiente. Sin embargo, en el mismo estudio, estos autores observaron que ante una alta relación de la profundidad de mezcla y la profundidad de la zona eufótica $\left(Z_{\text {mix }} / Z_{\text {eu }}>5\right)$, la morfología más eficiente corresponde a formas unicelulares grandes con aparato locomotor. Estos organismos logran explotar los recursos por encima del límite impuesto por las concentraciones de nutrientes (Naselli-Flores \& Barone, 2007) y en teoría, deberían beneficiarse de una irradiación alta y de una baja disponibilidad de nutrientes. Lo anterior concuerda con lo encontrado en el presente estudio e invita a indagar profundamente sobre cómo los tensores naturales y antrópicos inciden sobre el ambiente lumínico y la dinámica de los nutrientes.

\section{Conclusiones}

La comparación entre los estudios previos ( $\mathrm{mS}$ y $\mathrm{mT}$ ) y el último año de monitoreo (msENSO) muestra claramente dos aspectos relevantes que reflejan a través de los rasgos funcionales del fitoplancton el cambio de las condiciones de la ciénaga de Ayapel durante la franja de tiempo estudiada. El primero está asociado a la disminución del biovolumen y el segundo, al cambio de las estrategias morfológicas relacionadas específicamente con el cambio de la predominancia del GFBM III al V, que evidencia una adecuación de formas filamentosas a formas flageladas, así como el cambio de una estrategia ecológica $r$ a una primariamente adquisitiva como la $k$.

\section{Agradecimientos}

Los autores agradecen al grupo de investigación GeoLimna de la Universidad de Antioquia y a CORPOAYAPEL por el apoyo logístico y experimental en la realización de este estudio.

\section{Referencias}

Aguilera Díaz, M. M. (2009). Ciénaga de Ayapel: Riqueza en biodiversidad y recursos hídricos. Bogotá D.C.: Banco de la República de Colombia. https:/ / doi.org/10.32468/dtseru.112

Aguilera Díaz, M. M. (2011). La economía de las ciénagas del Caribe colombiano. Bogotá D.C.: Banco de la Republica de Colombia.

Aguirre, N., Palacio, J. A., Flórez, M. T., Wills, A., Caicedo, O., Jiménez, L. F. \& Vélez, J. L. (2005). Análisis de la relación río-ciénaga y su efecto sobre la producción pesquera en el sistema cenagoso de Ayapel, Colombia. (Informe técnico). Medellín, Colombia: Grupo de Investigación en Gestión y Modelación Ambiental.

Amorim, C. A., Valença, C. R., de Moura-Falcão, R. H. \& do Nascimento Moura, A. (2019). Seasonal variations of morpho-functional phytoplankton groups 
influence the top-down control of a cladoceran in a tropical hypereutrophic lake. Aquatic Ecology, 8, 1-12. https://doi.org/10.1007/s10452-019-09701-8

Bortolini, J. C., Moresco, G. A., De Paula, A. C. M., Jati, S. \& Rodrigues, L. C. (2016). Functional approach based on morphology as a model of phytoplankton variability in a subtropical floodplain lake: a longterm study. Hydrobiologia, 767(1), 151 - 163. https://doi.org/10.1007/s10750-015-2490-z

Comisión Económica para América Latina y el Caribe. -CEPAL- (2012). Valoración de daños y pérdidas. Ola invernal en Colombia 2010-2011. Bogotá D.C.: Misión BID.

Chaffin, J. D., Bridgeman, T. B., Heckathorn, S. A. \& Krause, A. E. (2012). Role of suspended sediments and mixing in reducing photoinhibition in the bloom-forming cyanobacterium Microcystis. Journal of Water Resource and Protection, 4(12), 1029 - 1041. https:/ / doi.org/10.4236/jwarp.2012.412119

Correa, M. V. (2013). La Mojana: tres años bajo el agua. https://www.elcolombiano.com/historico/la_mojana_tres_anos_bajo_el_agua-GCec_250521

Corporación Autónoma Regional de los Valles del Sinú y del San Jorge - CVS. (2014). Plan de acción para la temporada invernal en el departamento Córdoba. San Jorge, Colombia: Subdirección de gestión ambiental CVS, Grupo gestión del riesgo.

El Colombiano. (2016, September). S.O.S para salvar 160 Km2 de la ciénaga de Ayapel. https:/ / www. elcolombiano.com/colombia/sos-para-salvar-160km2-de-la-cienaga-de-ayapel-FE5042263

Esteves, F. A. (1998). Fundamentos de limnología. Rio de Janeiro: Interciencia Ltda.

Garcia, L., Marrugo, J. L. \& Alvis, E. M. (2010). Contaminación por mercurio en humanos y peces en el municipio de Ayapel, Córdoba, Colombia. Revista de la Facultad Nacional de Salud Pública, 28(2), 118-124.

Garduño, G., Oliva, M. G., Lugo, A., Mendoza, M. B., Quintanar, R. E. \& Conforti, V. (2011). Trachelomonas (Euglenophyta) from a eutrophic reservoir in Central Mexico. Journal of Environmental Biology, $32,463-471$.

Grime, J. (1977). Evidence for the existence of three primary strategies in plants and its relevance to ecological and evolutionary theory. The American Naturalist, 111(982), 1169-1194. https:/ / doi.org/10.1086/283244
Hernández, E., Aguirre, N. J., Palacio, J. A. \& Ramírez, J. J. (2008). Variación espacio temporal de la asociación fitoplanctónica en diferentes momentos del pulso hidrológico en la cienaga de Ayapel (Córdoba), Colombia. Actualidades Biológicas, 30(88), 67-81. Hernández-Atilano, E., Aguirre, N. J., Palacio, J., Ramírez-Restrepo, J., Duque, S., Guisande-González, C. \& Mogollón, M. (2012). Rasgos morfológicos del fitoplancton en seis sistemas leníticos de las regiones Amazónica, Andina y Caribe de Colombia. Actualidades Biológicas, 34(96), 67-83.

Hillebrand, H., Dürselen, C., Kirschtel, D., Pollingher, U. \& Zohary, T. (1999). Biovolume calculation for pelagic and benthic microalgae. Journal of Phycology, 35(2), 403-424. https://doi.org/10.1046/j.1529-8817.1999.3520403.x IDEAM. (2015). El Niño continúa presente y se intensifica. Recuperado de http:/ / www.ideam.gov.co/web/ Izaguirre, I., Allende, L., Escaray, R., Bustingorry, J., Pérez, G. \& Tell, G. (2012). Comparison of morpho-functional phytoplankton classifications in human-impacted shallow lakes with different stable states. Hydrobiologia, 698(1), 203 - 216. https:// doi.org/10.1007/s10750-012-1069-1

Jaramillo, J. C. \& Aguirre, N. J. (2012). Spatio-temporal changes in plankton in the Ciénaga de Ayapel (Córdoba-Colombia), during the period of lower water level. Caldasia, 34(1), 213 - 26.

Junk, W. J. \& Wantzen, K. M. (2004). The flood pulse concept: new aspects, approaches and applications - an update. Proceedings of the Second International Symposium on the Management of Large Rivers for Fisheries, 117 - 149.

Kruk, C., Huszar, V. L. M., Peeters, E. T. H. M., Bonilla, S., Costa, L., Lürling, M., Reynolds, C. \&Scheffer, M. (2010). A morphological classification capturing functional variation in phytoplankton. Freshwater Biology, 55(3), 614 627. https://doi.org/10.1111/j.1365-2427.2009.02298.x

Kruk, C., Peeters, E.T. H. M., Van Nes, E. H., Huszar, V. L. M., Costa, L. S. \& Scheffer, M. (2011). Phytoplankton community composition can be predicted best in terms of morphological groups. Limnology and Oceanography, 56(1), 110 - 118. https:// doi.org/10.4319/ lo.2011.56.1.0110

Kruk, C. \& Segura, A. M. (2012). The habitat template of phytoplankton morphology-based 
functional groups. Hydrobiologia, 698(1), 191 - 202. https://doi.org/10.1007/s10750-012-1072-6

Kruk, C., Devercelli, M., Huszar, V.L.M., Hernández, E., Beamud, G., Diaz, M., Silva, L.H.S. \& Segura, A.M. (2017). Classification of Reynolds phytoplankton functional groups using individual traits and machine learning techniques. Freshwater Biology, 62(10), 16811692. https:/ /doi.org/10.1111/fwb.12968

Lins, R. P. M., Ovruski de Ceballos, B. S., Serramo Lopez, L. C. \& Gomes Barbosa, L. (2017). Phytoplankton functional groups in a tropical reservoir in the Brazilian semiarid región. Revista de Biología Tropical, 65(3), 1129 - 1141. https://doi.org/10.15517/rbt.v65i3.29446

Margalef, R. (1993). Limnología. Barcelona: Editorial Omega

Mihaljevic, M., Stevic, F., Spoljaric, D. \& Zuna pfeiffer, T. (2015). Spatial pattern of phytoplankton based on the Morphology- Based Functional approach along a river floodplain gradient. River Research and Applications, 31(2), 228 - 238. https:/ / doi.org/10.1002/ rra.2739

Mur, R. L., Skulberg, O. M. \& Utkilen, H. (1999). Cyanobacteria in the Environment. En: Chorus, I. \& Bartram, J. (Eds). Toxic Cyanobacteria in Water: A guide to their public health consequences.

Muñoz-López, C. L., Aranguren-Riaño, N. J. \& Duque, S. R. (2017). Functional morphology of phytoplankton in a tropical high mountain lake: Tota Lake (BoyacáColombia). Revista de Biología Tropical, 65(2), 669 - 684. https://doi.org/10.15517/rbt.v65i2.23903

Naselli-Flores, L. \& Barone, R. (2007). Pluriannual morphological variability of phytoplankton in a highly productive mediterranean reservoir (Lake Arancio, Southwestern Sicily). Hydrobiologia, 578(1), 87 - 95. https:// doi.org/10.1007/s10750-006-0436-1

Naselli-Flores, L., Padisák, J. \& Albay, M. (2007). Shape and size in phytoplankton ecology: Do they matter? Hydrobiologia, 578(1), 157 - 161. https:/ / doi.org/10.1007/s10750-006-2815-z

O'Farrell, I., Tell, G. \& Podlejski, A. (2001). Morphological variability of Aulacoseira granulata (Ehr.) Simonsen (Bacillariophyceae) in the Lower Paraná River (Argentina). Limnology, 2(2), 65 - 71. https://doi.org/10.1007/s102010170001

Oda, A. C. R. \& Bicudo, C. E. M. (2006). Ecology of Peridinium gatunense and Peridinium umbonatum
(Dinophyceae) in a shallow tropical oligotrophic reservoir (IAG Pond), São Paulo, southeast Brazil. Acta Limnologica Brasiliensia, 18(2), 165 - 180.

Pacheco, J. P., Iglesias, C., Meerhoff, M., Fosalba, C., Goyenola, G., Teixeira-de Mello, F., García, S., Gelós, M. \& García-Rodrígues, F. (2010). Phytoplankton community structure in five subtropical shallow lakes with different trophic status (Uruguay): a morphology-based approach. Hydrobiologia, 646(1), 187-197. https:// doi.org/10.1007/s10750-010-0180-4

Roldán, G. \& Ramírez, J. J. (2008). Fundamentos de limnologia neotropical. Medellín, Colombia: Universidad de Antioquia.

Rangel-Ch, J. O. \& Arellano, H. (2010). Diversidad Biótica IX: Ciénagas de Córdoba. Biodiversidad, Ecología y Manejo ambiental. En: Rangel-Ch., J.O. (ed). Clima. Bogotá D.C.: Instituto de Ciencias Naturales.

Reynolds, C. S. (1980). Phytoplankton assemblages and their periodicity in stratifying lake systems. Ecography, 3(3), 141-159.

https:// doi.org/10.1111/j.1600-0587.1980.tb00721.x Reynolds, C. S. (1984). The ecology of freshwater phytoplankton. Cambridge: Cambridge University Press. Reynolds, C. S. (1997). Vegetation processes in the pelagic: a model for ecosystem theory. The quarterly review of biology, 73(2), 228. https://doi.org/10.1086/420250

Reynolds, C. S., Huszar, V., Kruk, C., NaselliFlores, L. \& Melo, S. (2002). Towards a functional classification of the freshwater phytoplankton. Journal of Plankton Research, 24(5), 417 - 428. https:// doi.org/10.1093/plankt/24.5.417

Segura, A. M., Sarthou, F. \& Kruk, C. (2018). Morphology-based differences in the thermal response of freshwater phytoplankton. Biology Letters, 14(5), 1 - 4. https://doi.org/10.1098/rsbl.2017.0790

Utermohl, H. (1958). Zur Vervollkommnung der quantitativen phytoplancton-methodik. Verhandlungen des Internationalen Verein Limnologie, 9, 1 - 38. https://doi.org/10.1080/05384680.1958.11904091

Vollenweider, R. A. (1968). Scientific fundamentals of the eutrophication of lakes and flowing water, with particular reference to phosphorus and nitrogen as factors in eutrophication. (Tech. Rep). Paris, Francia: OECD. 159 pp. 
Wurts, W. A. \& Durborow, R. M. (1992). Interactions of pH, carbon dioxide, alkalinity and hardness in fish ponds. Ames, USA: Southern Regional Aquaculture Center Publication, Iowa State University.
Zapata, G., Bermúdez, J. G., Rodríguez, G. \& Arango, M. I. (2013). Cartografía Geológica de la Plancha 83 Nechí (Departamento de Antioquia). Bogotá D.C.: Ministerio de Minas y Energía-Servicio Geológico Colombiano. 
Ana María Zabala Agudelo

Universidad de Antioquia

Medellín, Colombia

ana.zabala@udea.edu.co

https://orcid.org/0000-0002-7424-8936

\section{Esnedy Hernández}

Universidad de Antioquia

Medellín, Colombia

esnedy.hernandez@udea.edu.co

https://orcid.org/0000-0003-2380-1436

\section{Diana María Agudelo}

Universidad de Antioquia

Medellín, Colombia

diana.agudeloe@udea.edu.co

https://orcid.org/0000-0003-2068-2902

\section{Nestor Jaime Aguirre}

Universidad de Antioquia

Medellín, Colombia

nestor.aguirre@udea.edu.co

https://orcid.org/0000-0003-0047-3807

\section{Fabio de Jesús Vélez}

Universidad de Antioquia

Medellín, Colombia

fabio.velez@udea.edu.co

https://orcid.org/0000-0001-6348-6405
Variación temporal de la morfología funcional del fitoplancton en una planicie inundable del Caribe Colombiano

Citación del artículo: Zabala, A.A., Henández, E., Agudelo, D.A., Agurre, N.J. \& Vélez, F.J. (2019). Variación temporal de la morfología funcional del fitoplancton en una planicie inundable del caribe Colombiano. Biota Colombiana, 20(2), 2-19. DOI: 10.21068/c2019.v20n02a01.

Recibido: 2 de septiembre de 2018

Aceptado: 29 de julio de 2019 